\title{
Study on the Equivalence Between Nonlinear Least Squares Method and Correlation Method in Period Extraction of Torsion Pendulum
}

\author{
Wenze Zhan ${ }^{a^{*}}$, Weihuang $\mathrm{Wu}^{\mathrm{b}}$ \\ School of Mechanical Engineering and Electronic Information, China University of Geosciences, \\ Wuhan 430074, China \\ azhanwenze@163.com, bwuweihuangthanks@163.com
}

Keywords: period extraction; nonlinear least squares fitting method; correlation method; Gaussian white noise; accuracy

\begin{abstract}
In the measurement of $G$ using time-of-swing method, the accurate determination of $G$ depends on the period extraction of torsion balance with high precision. There are a great many methods of extracting period, and the nonlinear least squares fitting method and the correlation method are most frequently applied to extract the torsion balance period accurately. Though some papers have claimed the two methods are equivalent, lacking in complete theoretical derivation. In this paper, the influence of Gaussian white noise on the period extraction accuracy by the two methods is theoretically derived detailedly and simulated in MATLAB. Both results show that although there exists some differences between two methods in process of data analysis, the accuracy of period extraction is still the same.
\end{abstract}

\section{Introduction}

The period extraction of a torsion pendulum with high precision is of great importance and necessity to some gravitational experiments. For example, in the measurement of the Newtonian gravitational constant $G$ with the time-of-swing method, one needs to estimate the period of the torsion balance with high precision[1,2,3,4]. However, the experimental data is inevitably polluted by a variety of noises, and thus the precision of period estimation may be also limited by these noises. The Gaussian white noise is a kind of ideal mathematical model and denotes some random noise sequences, for example, the environment noise can be viewed as the stochastic noise sequence[5]. Maybe the white noise is not a perfect model for the torsion pendulum experiments, but it is useful and advisable for theoretical analysis to judge a method is feasible or not[6]. Hence, the influence of the Gaussian white noise on the period estimation in measurement of $G$ is still well worth studying.

To date, there are different varieties of methods to extract the period of torsion pendulum. Based on the data processing domain, they could be divided into two categories: one is to analyze the spectrum or power spectral density in frequency domain, such as the Fast Fourier Transform (FFT) method[7]; the other kind is fitting in time domain, mainly including the 'all-poles' method[8], the nonlinear least squares fitting method[9,10,11], and the correlation method[12,13]. The FFT method is used to extract the signal frequency by finding the peak of the frequency spectrum, and it is simple to program and fast to perform, but meantime the interested signal frequency is easily disturbed by other frequency signal components. Another disadvantage of the FFT method is that the window function could cause the spectrum leakage. The 'all-poles' method is to perform a linear fitting on the sequence of time and pole values. It is simple to implement and easy to calculate, but only uses the extreme values of the experimental data to obtain information, which is a great waste to the experimental data. Meanwhile, since the experimental data is influenced by many disturbance factors, such as various noises and the damping effect, the period extraction is naturally subject to a significant deviation.

For the nonlinear least squares fitting method, once the residual error between the fitting results and experimental data is minimum, then we can obtain the best estimate of the period. Obviously, selecting an appropriate objective function is of central essence to estimate period by this method. The correlation method is based on the orthogonal principle of trigonometric function, which only 
focus on period, and then extract period by analyzing the change of the phase. This method is not only highly efficient and accurate, but also insensitive to higher harmonic, the damping effect and the slow drift of period. Though some papers have claimed the two methods are equivalent, lacking in complete theoretical derivation[14].

In this paper, a sinusoidal signal model with the Gaussian white noise is established, and the influences of white noise on the accuracy of period estimation by the nonlinear least squares fitting method and the correlation method is theoretically derived in detail. It turns out that the influences of the Gaussian white noise on the accuracy of period estimation by the two methods are the same with each other. Then, we use the two methods to extract the period of torsion pendulum respectively by performing simulation in the software MATLAB. The results indicate that both the two methods could obtain periods with high precision, which are in good agreement with respective uncertainties, and though there exist some differences between the two methods in the process of data analysis, the accuracy of the period estimation is still the same.

\section{The nonlinear least squares fitting method for period estimation}

The nonlinear least squares fitting method is one of traditional methods for data analysis, which can obtain the amplitude, period and phase at the same time. Firstly, we should chooses an appropriate objective function to fit the experimental data. Then, when the residual error between the fitting results and the experimental data is minimum, we can get the best estimates of the signal parameters [15]. Generally, the deflection angle of a torsion balance could be modeled as a sinusoidal signal with the Gaussian white noise, expressed by

$$
y_{i}=\bar{y}_{i}+\varepsilon_{i}=\theta_{0} \cos \left(\omega_{0} t_{i}+\phi\right)+\varepsilon_{i},
$$

where $\bar{y}_{i}$ is the real deflection angle of a torsion balance, $\varepsilon_{i}$ is the Gaussian white noise sequence, $\theta_{0}$ is the amplitude of the signal, $\omega_{0}$ is the angular frequency, and $\emptyset$ is the initial phase of the signal. The statistical properties of the Gaussian white noise is: $\mathrm{E}\left[\varepsilon_{i}\right]=0, \operatorname{cov}\left[\varepsilon_{i}, \varepsilon_{j}\right]=\delta_{i j} \sigma^{2}, \sigma$ is the amplitude of the noise.

The objective function is chosen as

$$
\tilde{y}_{i}=\tilde{\theta}_{0} \cos \left(\tilde{\omega}_{0} t_{i}+\tilde{\phi}\right)
$$

with the fitting parameters $\tilde{\theta}_{0}, \widetilde{\omega}_{0}$ and $\emptyset$. Hence, the residual error between the fitting values and the sampling values of the deflection angle is expressed as follows:

$$
\sigma_{f i t}\left(\tilde{\theta}_{0}, \tilde{\omega}_{0}, \tilde{\phi}\right)=\sqrt{\sum_{i=1}^{N}\left(y_{i}-\tilde{y}_{i}\right)^{2} / N}
$$

The nonlinear least squares fitting method selects the residual erroe $\sigma_{f i t}$ as a criterion to determine the three best estimators $\tilde{\theta}_{0}, \widetilde{\omega}_{0}$ and $\emptyset$. When the three parameters satisfy the three equations as $\partial \sigma_{f i t} /$ $\partial \tilde{\theta}_{0}=0, \partial \sigma_{f i t} / \partial \tilde{\omega}_{0}=0$, and $\partial \sigma_{f i t} / \partial \tilde{\phi}=0$, namely:

$$
\left\{\begin{array}{l}
\sum_{i=1}^{N} \cos \left(\tilde{\omega}_{0} t_{i}+\tilde{\phi}\right)\left[y_{i}-\tilde{\theta}_{0} \cos \left(\tilde{\omega}_{0} t_{i}+\tilde{\phi}\right)\right]=0 \\
\sum_{i=1}^{N} t_{i} \sin \left(\tilde{\omega}_{0} t_{i}+\tilde{\phi}\right)\left[y_{i}-\tilde{\theta}_{0} \cos \left(\tilde{\omega}_{0} t_{i}+\tilde{\phi}\right)\right]=0, \\
\sum_{i=1}^{N} \sin \left(\tilde{\omega}_{0} t_{i}+\tilde{\phi}\right)\left[y_{i}-\tilde{\theta}_{0} \cos \left(\tilde{\omega}_{0} t_{i}+\tilde{\phi}\right)\right]=0
\end{array}\right.
$$

the obtained three parameter is the best estimates consequently. In factual experiments, the noise sequence is usually very small, thus $\left|\varepsilon_{i}\right|<<\theta_{0}$. For the three parameters, their values are functions of $\left\{y_{i}\right\}$ and expressed approximately as 


$$
\left\{\begin{array}{c}
\tilde{\omega}_{0}\left\{y_{i}\right\}=\tilde{\omega}_{0}\left\{\bar{y}_{i}+\varepsilon_{i}\right\} \approx \tilde{\omega}_{0}\left\{\bar{y}_{i}\right\}+\sum_{j=1}^{N} \tilde{\omega}_{i, j} \varepsilon_{j}=\omega_{0}+\sum_{j=1}^{N} \tilde{\omega}_{i, j} \varepsilon_{j} \\
\tilde{\theta}_{0}\left\{y_{i}\right\}=\tilde{\theta}_{0}\left\{\bar{y}_{i}+\varepsilon_{i}\right\} \approx \tilde{\theta}_{0}\left\{\bar{y}_{i}\right\}+\sum_{j=1}^{N} \tilde{\theta}_{i, j} \varepsilon_{j}=\theta_{0}+\sum_{j=1}^{N} \tilde{\theta}_{i, j} \varepsilon_{j} \\
\tilde{\phi}\left\{y_{i}\right\}=\tilde{\phi}\left\{\bar{y}_{i}+\varepsilon_{i}\right\} \approx \tilde{\phi}\left\{\bar{y}_{i}\right\}+\sum_{j=1}^{N} \tilde{\phi}_{i, j} \varepsilon_{j}=\phi+\sum_{j=1}^{N} \tilde{\phi}_{i, j} \varepsilon_{j}
\end{array}\right.
$$

where $\widetilde{\omega}_{i, j}=\partial \widetilde{\omega}_{0}\left\{y_{i}\right\} / \partial y_{j}$. The residual error between $\widetilde{\omega}_{0}\left\{y_{i}\right\}$ and $\omega_{0}$ depends on $\varepsilon_{i}$. It is obvious that $\widetilde{\omega}_{0}\left\{y_{i}\right\}=\omega_{0}$, and if the response of noise verge to to zero. $\widetilde{\theta}_{i, j}$ and $\widetilde{\emptyset}_{i, j}$ have the similar meaning.

Substituting Eq.(5) to $y_{i}$, with $\omega_{0}=2 \pi / \tau_{0}, t=m \tau_{0}$ and $\mathrm{m} \gg>1$, we obtain the liner equations for $\widetilde{\omega}_{i, j}, \tilde{\theta}_{i, j}$ and $\widetilde{\emptyset}_{i, j}$ as

$$
\frac{\tau_{0}}{\Delta t}\left[\begin{array}{ccc}
-\frac{m}{2} & -\frac{\cos (2 \phi)}{8 \pi} & 0 \\
\frac{\cos (2 \phi)}{8 \pi} & \frac{m}{6}-\frac{\sin (2 \phi)}{8 \pi} & \frac{m}{4}-\frac{\sin (2 \phi)}{8 \pi} \\
0 & \frac{m}{4}-\frac{\sin (2 \phi)}{8 \pi} & \frac{m}{2}
\end{array}\right]\left[\begin{array}{c}
\tilde{\theta}_{i, j} \\
t \theta_{0} \tilde{\omega}_{i, j} \\
\theta_{0} \tilde{\phi}_{i, j}
\end{array}\right]=-\left[\begin{array}{c}
\cos \left(\omega_{0} t_{j}+\phi\right) \\
\frac{t_{j}}{t} \sin \left(\omega_{0} t_{j}+\phi\right) \\
\sin \left(\omega_{0} t_{j}+\phi\right)
\end{array}\right] .
$$

The matrix on the left side in Eq.(6) is remained to the order of $o\left(m^{0}\right)$. Thus, $\widetilde{\omega}_{i, j}, \tilde{\theta}_{i, j}$ and $\widetilde{\emptyset}_{i, j}$ are obtained, where the interested $\widetilde{\omega}_{i, j}$ is expressed as

$$
\tilde{\omega}_{i, j}=\frac{\Delta t}{t m \theta_{0} \tau_{0}}\left[\left(12-\frac{24 t_{j}}{t}-\frac{6 \sin (2 \phi)}{\pi m}\right) \sin \left(\omega_{0} t_{j}+\phi\right)-\frac{6}{\pi m} \cos (2 \phi) \cos \left(\omega_{0} t_{j}+\phi\right)\right] .
$$

The residula error of the estimate $\widetilde{\omega}_{0}$ is

$$
\sigma_{\tilde{w}}=\sqrt{E\left(\sum_{j=1}^{N} \tilde{\omega}_{i, j} \varepsilon_{i}\right)^{2}}
$$

In general, we choose the phase $|\varnothing|<<1$. The sampling interval is $\Delta t$, the number of the experimental data is $N$, then the sampling time sequence is $t_{j}=j \Delta t$, and the length of time is $t=N \Delta t$. The sum under the root sign can be converted into the integral, expressed as

$$
\sum_{j=1}^{N} \tilde{\omega}_{i, j} \varepsilon_{i} \approx \frac{12}{\theta_{0} t^{2}} \int_{0}^{t}\left(1-\frac{2 x}{t}\right) \sin \left(\omega_{0} x\right) \varepsilon(x) d x .
$$

For Eq.(9), with $t=m \tau_{0}$, remained to the order of $o\left(\mathrm{~m}^{-3}\right)$, the residual errpr of $\widetilde{\omega}_{0}$ is further expressed as

$$
E\left(\sum_{j=1}^{N} \tilde{\omega}_{i, j} \varepsilon_{i}\right)^{2}=\left(\frac{12}{\theta_{0} t^{2}}\right)^{2} \sigma^{2} \Delta t \int_{0}^{t}\left(1-\frac{2 x}{t}\right)^{2} \sin ^{2}\left(\omega_{0} x\right) d x=\frac{72 \sigma^{2} \Delta t}{\theta_{0}{ }^{2} t^{4}}\left(\frac{1}{3} t-\frac{2 t}{\omega_{0}{ }^{2}}\right) \approx \frac{24 \sigma^{2} \Delta t}{\theta_{0}{ }^{2} m^{3} \tau_{0}^{3}} .
$$

The uncertainty of frequency caused by the white noise is

$$
\sigma_{\tilde{w}}=\sqrt{E\left(\sum_{j=1}^{N} \tilde{\omega}_{i, j} \varepsilon_{i}\right)^{2}} \approx \sqrt{\frac{24 \sigma^{2} \Delta t}{\theta_{0}^{2} m^{3} \tau_{0}^{3}}} .
$$

Then the relative uncertainty of period caused by the white noise is

$$
\frac{\sigma_{\tau}}{\tau_{0}} \sim \frac{\sigma_{\tilde{w}}}{\omega_{0}}=\frac{\sqrt{6}}{\pi} \frac{\sigma}{\theta_{0} m} \sqrt{\frac{\Delta t}{m \tau_{0}}} .
$$

\section{The correlation method for period estimation}

The correlation method, also named the phase method, is a highly efficient and precise method for subtle signal analysis, and usually used in some engineering fields such as in global positioning systems, seismology and ultrasonic[12]. It could effectively restrain the higher harmonic components 
and nonlinear effect, and not sensitive to the linear drift of the equilibrium position. Generally, the deflection angle of the sinusoidal signal model with white noise can be also expressed as

$$
y_{i}=\theta_{0} \cos \left(\omega_{0} t_{i}+\phi\right)+\varepsilon_{i}=a \cos \left(\omega_{0} t_{i}\right)+b \sin \left(\omega_{0} t_{i}\right)+\varepsilon_{i}
$$

where $a$ and $b$ are the decomposed coefficients of $\theta_{0}$, and $\tan ^{-1}(b / a)$ represents the initial phase $\emptyset$. Firstly, we choose a strictly sinusoidal function $\cos \left(\omega_{\text {ref }} t\right)$ as the reference signal with $\omega_{\text {ref }} \approx \omega_{0}$ and period $\tau_{\text {ref }}=2 \pi / \omega_{\text {ref }}$, then compare the sampling data $\left\{y_{i}\right\}$ with the strictly sinusoidal function, and calculate the phase angle $\emptyset_{j}$ at the $j$ th period. The phase angle is determined by the cross-correlation function of the reference signal and the sampling data, expressed as

$$
\phi_{j}=\tan ^{-1} \frac{b_{j}}{a_{j}}=\tan ^{-1} \frac{\frac{2}{\tau_{r e f}} \int_{j \tau_{r e f}}^{(j+1) \tau_{r e f}} y(t) \sin \left(\omega_{r e f} t\right) d t}{\frac{2}{\tau_{r e f}} \int_{j \tau_{r e f}}^{(j+1) \tau_{r e f}} y(t) \cos \left(\omega_{r e f} t\right) d t} .
$$

In order to keep the phase unambiguous, the frequency $\omega_{\text {ref }}$ of the reference signal should be very closed to the real frequency $\omega_{0}$. Generally, we choose the initial phase $\tan ^{-1}(b / a) \ll 1$, then $\Delta \emptyset_{j}$ can be further expressed as approximately

$$
\Delta \phi_{j}=-\phi_{j} \approx \phi+2 \pi \frac{\omega_{r e f}-\omega_{0}}{\omega_{r e f}} j+\delta \phi_{j},
$$

where $\varnothing$ is the initial phase, and $\delta \emptyset_{j}$ is a random variable dependent on the Gaussian white noise $\varepsilon_{i}$. Due to $\tau_{\text {ref }} \approx \tau_{0}, \delta \emptyset_{j}$ can be written as

$$
\delta \phi_{j} \approx \frac{2}{\tau_{0} \theta_{0}} \int_{j \tau_{0}}^{(j+1) \tau_{0}} \varepsilon(t) \sin \left(\omega_{0} t\right) d t
$$

The linear fitting of Eq.(15) gives the estimate of period as

$$
\tau_{0}=\tau_{\text {ref }}+\frac{K}{2 \pi} \tau_{\text {ref }}
$$

where $k$ is the estimate for the slope of Eq.(15). According to Eq.(15), the estimates of the intercept and the slope are

$$
\left(\begin{array}{l}
\hat{\phi} \\
K
\end{array}\right)=\left(C^{T} C\right)^{-1} C^{T} B=\left(\begin{array}{cc}
\frac{4 m-2}{m(m+1)} & \frac{-6}{m(m+1)} \\
\frac{-6}{m(m+1)} & \frac{-12}{m\left(1-m^{2}\right)}
\end{array}\right)\left(\begin{array}{l}
\sum_{i=0}^{m-1} \Delta \phi_{i} \\
\sum_{i=0}^{m-1} i \Delta \phi_{i}
\end{array}\right) .
$$

$\Delta k$ is the error of lope, which is caused by the phase noise $\delta \emptyset_{j}$ and it can be expressed as

$$
\Delta K \approx \frac{6}{m^{2}}\left(-1 \frac{2}{m}\right)\left(\begin{array}{l}
\sum_{i=0}^{m-1} \delta \phi_{i} \\
\sum_{i=0}^{m-1} i \delta \phi_{i}
\end{array}\right)=\frac{6}{m^{2}} \sum_{i=0}^{m-1}\left(\frac{2 i}{m}-1\right) \delta \phi_{i} .
$$

Using the standard deviation to express the deviation of the slope is

$$
\sigma(\Delta \hat{K})=\sqrt{E\left[(\Delta \hat{K})^{2}\right]}=\sqrt{\frac{24 \sigma^{2} \Delta t}{\theta_{0}^{2} \tau_{0} m^{3}}} .
$$

Then the uncertainty of period caused by the Gaussian white noise is

$$
\frac{\sigma_{\tau}}{\tau_{0}} \sim \frac{\sigma_{\tilde{w}}}{\omega_{0}}=\frac{\omega_{r e f}}{2 \pi} \sigma(\Delta \hat{K}) \frac{1}{\omega_{0}}=\frac{\sqrt{6}}{\pi} \frac{\sigma}{\theta_{0} m} \sqrt{\frac{\Delta t}{m \tau_{0}}} .
$$

Obviously, the Eq.(12) and Eq.(21) are the same to each other, that is to say the uncertainties of the Gaussian white noise on the period by the two methods are the same in theory, which can be further expressed as approximately 


$$
\sigma_{\tau} \approx \frac{\sigma_{\tilde{w}}}{\omega_{0}} \tau_{0} \frac{\sqrt{6}}{\pi} \frac{\sigma \tau_{0}}{\theta_{0} m} \sqrt{\frac{\Delta t}{m \tau_{0}}}
$$

\section{Results and discussions}

The simulation by MATLAB is performed to compare the nonlinear least squares fitting method and the correlation method in extracting the period of torsion pendulum. The simulated data is generated by the model Eq.(1). Based on the real experimental data[6], we set the parameters of the simulation as $\theta_{0}=0.0015 \mathrm{rad}, \tau_{0}=537.024385 \mathrm{~s}, \varnothing \omega_{0}=0.0117 \mathrm{rad} / \mathrm{s}, \emptyset=0.002 \mathrm{rad}$, the amplitude of the Gaussian white noise as the Table 1 shows, the sampling frequency $f_{s}=2 \mathrm{~Hz}$, the number of the sampling data $N=500000$. In order to ensure the reliability of the estimated results, a large number of statistical experiments are needed and then the statistical average value of the test results is used to give the estimate. We counted 500 times of the experimental results and obtained the simulation results in terms of different noise intensity, which is shown in table 1 with signal to noise ratio.

Table 1 The period fitting results under different noise intensity (the period is set as 537.024385s)

\begin{tabular}{|c|c|c|c|c|}
\hline \multirow[b]{2}{*}{$\begin{array}{l}\text { Different noise } \\
\text { intensity }\end{array}$} & \multicolumn{2}{|c|}{ Period value (s) } & \multicolumn{2}{|c|}{ Uncertainty of period(s) } \\
\hline & $\begin{array}{l}\text { Least squares } \\
\text { fitting }\end{array}$ & $\begin{array}{c}\text { Correlation } \\
\text { method }\end{array}$ & $\begin{array}{c}\text { Least squares } \\
\text { fitting }\end{array}$ & $\begin{array}{c}\text { Correlation } \\
\text { method }\end{array}$ \\
\hline 0.001 & 537.024385 & 537.024385 & 0.000001 & 0.000001 \\
\hline 0.05 & 537.024385 & 537.024385 & 0.000007 & 0.000006 \\
\hline 0.01 & 537.024385 & 537.024385 & 0.000013 & 0.000013 \\
\hline 0.05 & 537.024386 & 537.024386 & 0.000066 & 0.000062 \\
\hline 0.1 & 537.024386 & 537.024392 & 0.000133 & 0.000126 \\
\hline
\end{tabular}

Through the above simulation results, we can see that the periods extracted by the two methods are in good agreement within respective uncertanties and the given period $\tau_{0}$ belongs to the obtained period ranges of the two methods; namely, both the nonlinear least squares fitting method and the correlation method can obtain right and accurate periods of torsion balance, and the two methods are equivalent to each other in extracting the period. The Fig.1 shows the relation between frequency and residual error in the nonlinear least squares fitting method. Once the residual error is up to minimum, the obtained frequency is the best unbiased estimate. The Fig. 2 shows the relation between the deviation of phase and period in the correlation method. Once the slope of the fitting line is determined, the period can be obtained.

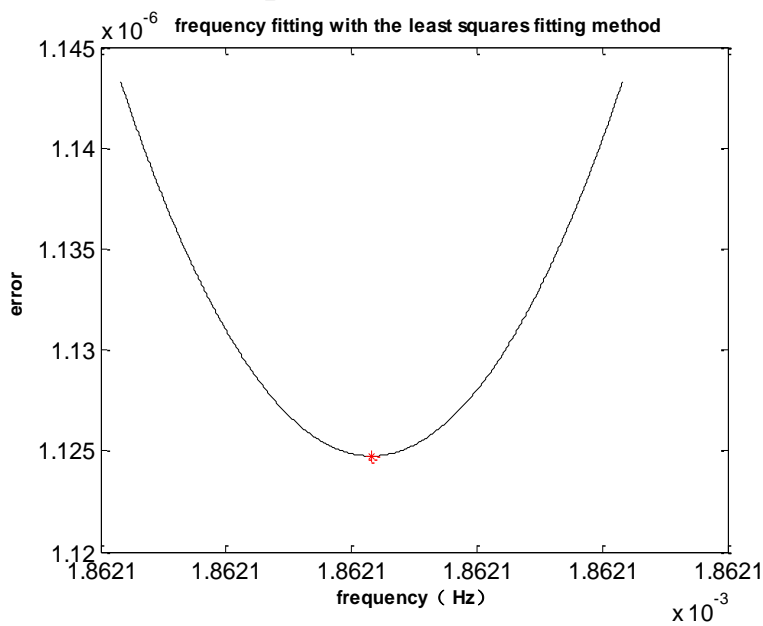

Fig. 1 The relation between frequency and residual error in the nonlinear least squares fitting method

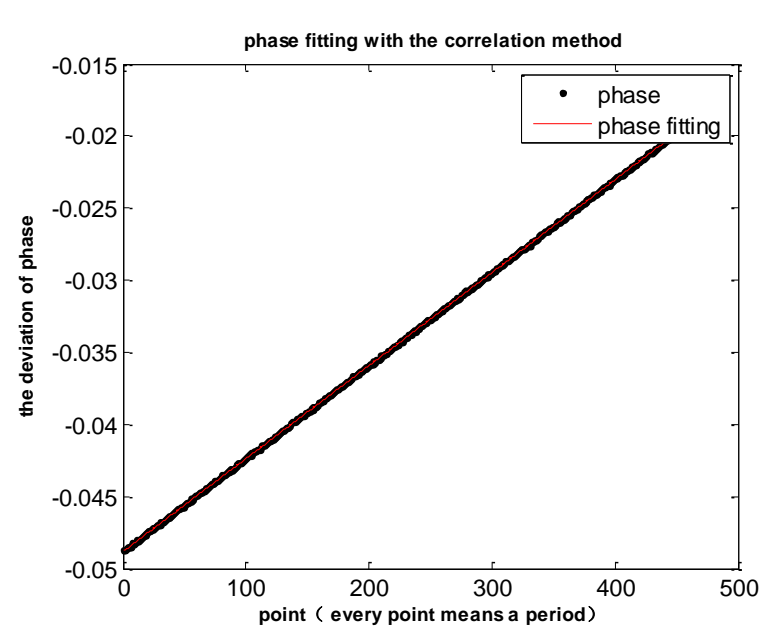

Fig. 2 The difference of phase in each period in the correlation method 


\section{Conclusions}

In conclusion, we have discussed the influence of the Gaussian white noise on the period estimation by the nonlinear least squares fitting method and the correlation method in detail. Both the theoretical analysis and simulation results show that these two methods could extract the period of torsion balance with high precision. Though there exist some differences between the two methods in the process of data analysis, the accuracy of the period estimation is the same. The nonlinear least squares fitting method can obtain the amplitude, period and phase at the same time, but in the occasion of a large amount of experimental data, the computing time will be longer with low efficiency. At same time, the objective function is of central essence to estimate period by this method. The correlation method only focuses on the period, which is is not only highly efficient and accurate, but also insensitive to higher harmonic, the damping effect and the slow drift of period. For the period estimation of torsion balance, much more interferences should be considered to improve the accuracy, such as the length of correlation time of noise, the signal model of the real signal in experiment, the influences of higher harmonic and so on.

\section{References}

[1]J. Luo, Z. K. Hu, Classical Quantum Gravity, 17, 2351.(2000)

[2]P. R. Hely: J Res Nat Bur Stand(US), 29:1-31. (1942)

[3]J. Luo, Q. Liu and L. C. Tu et al, Phys. Rev. Lett.,102(24):240801.( 2009)

[4]Q. Li. Research of systematic errors in G measurement with time-of-swing method[D]. Wuhan: Huazhong University of Science \& Technology, (2013) (In Chinese)

[5]Y. L. Tian, Y. Tu and C. G. Shao: Rev. Sci. Instrum.75,6.(2004)

[6] J. Luo, Y. Tian, C. G Shao, and D. H. Wang, Chin. Phys. B 24, 030401 (2015).

[7]C. E. Goldblum and R. C. Ritter, Rev. Sci. Instrum., 59,778.(1988)

[8]J. J. Snyder: Appl. Opt. 19, 1224-6.(1980)

[9]G. T. Gillies and T. C. Ritter: Rev. Sci. Instrum.64, 283.(1993)

[10]C. G. Shao, E. J. Luan and J. Luo: Rev. Sci. Instrum. 74, 2849.(2003)

[11]S. Yu, B. Yan and G. J. Chen: Journal of electronic measurement and instrument, 14(2).(2000) (In Chinese)

[12]J. S. Bendat and A. G. Piersol, Random Data: Analysis and Measurement Procedures[M]. Wiley, New York (1971)

[13]H. Li and Y. F. Wang: Journal of electronics \& information technology,32(3).(2010) (In Chinese)

[14] Luo J, Shao C G and Wang D H. Thermal noise limit on the period of a torsion pendulum [J].

Class. Quantum Grav. 26,195005, (2009)

[15]Y. T. Fei: Error theory and data processing[M]. Beijing: China Machine Press.(2008) (In Chinese) 